\title{
The protective effect of Thai Herbal Sahatsatara formula against white matter injury after chronic cerebral hypoperfusion in middle-aged rats
}

\author{
Saruda Kuraeiad ${ }^{\mathrm{a}}$, Anchalee Vattarakorn ${ }^{\mathrm{a}}$, Onusa Thamsermsang ${ }^{\mathrm{b}}$, Pravit Akarasereenont ${ }^{\mathrm{b}, \mathrm{c}}$, \\ Sompol Tapechum ${ }^{\mathrm{a}}$, Kanokwan Tilokskulchai ${ }^{\mathrm{a}}$, Narawut Pakaprot ${ }^{\mathrm{a}, *}$ \\ a Department of Physiology, Faculty of Medicine Siriraj Hospital, Mahidol University, Bangkok 10700 \\ Thailand \\ b Center of Applied Thai Traditional Medicine, Faculty of Medicine Siriraj Hospital, Mahidol University, \\ Bangkok 10700 Thailand \\ c Department of Pharmacology, Faculty of Medicine Siriraj Hospital, Mahidol University, Bangkok 10700 \\ Thailand
}

*Corresponding author, e-mail: narawut.pak@mahidol.ac.th

Received 9 Apr 2020

Accepted 7 Aug 2020

\begin{abstract}
Permanent bilateral common carotid artery occlusion (2VO) in rodents induces chronic cerebral hypoperfusion, mimicking vascular dementia in elderly people. It causes inflammation and oxidative stress, leading to neuronal loss in the hippocampus, white matter injuries and impairments of spatial learning and memory. Thai Herbal Sahatsatara formula (STF) has anti-inflammatory and antioxidant actions which might protect neurons and white matter. This experiment was to investigate the possible protective effects of STF. Twenty-eight middle-aged male Wistar rats (12 months old) were divided into 4 groups: Sham+sterile water (SW), 2VO+SW, 2VO+STF300 and 2VO+STF1000. All animals daily received either SW, STF $300 \mathrm{mg} / \mathrm{kg}$ or STF $1000 \mathrm{mg} / \mathrm{kg}$ orally after 2VO induction until the end of the experiment. Spatial learning and memory test were examined after 2VO induction for 60 days whereas the numbers of hippocampal neurons and white matter changes in the corpus callosum were investigated after the end of the behavioral test. Chronic cerebral hypoperfusion significantly caused spatial learning and memory deficits and white matter injuries in the corpus callosum while the numbers of hippocampal neurons were not significantly affected. STF (1000 mg/kg) attenuated the impairments of spatial learning and learning flexibility and white matter injuries. This is the first study to demonstrate the ability of Sahatsatara formula to attenuate spatial learning and learning flexibility impairments after chronic cerebral hypoperfusion by protecting the white matter in the corpus callosum. The results support the potential application of Sahatsatara formula against chronic cerebral hypoperfusion.
\end{abstract}

KEYWORDS: 2VO, cerebral hypoperfusion, hippocampus, Sahatsatara formula, white matter injury

\section{INTRODUCTION}

Cognitive impairments in executive function, episodic memory and processing speed are usually observed at an advanced age. Chronic cerebral hypoperfusion is one of many causes, resulting in cognitive impairments in elderly population. Many factors cause chronic cerebral hypoperfusion such as hypertension and diabetes. In non-invasive scanning study, the regional cerebral blood flow $(\mathrm{CBF})$ in the hippocampus, an area susceptible to hypoperfusion and important for learning and memory, was dramatically decreased in the vascular dementia patients [1]. The brains of aged adults who had cerebrovascular disease also showed cerebral white matter lesions which might be the result of chronic cerebral hypoperfusion [2]. There were associations between cognitive decline and white matter lesions in the elderly people [3].

Permanent bilateral common carotid artery occlusion (2VO) is a classical technique in rodents to mimic neurodegeneration caused by chronic cerebral hypoperfusion in ageing [4-6]. The 2VO model caused moderate global cerebral ischemia. The decrease of $\mathrm{CBF}$ in $2 \mathrm{VO}$ model was approximately $40-60 \%$ of the normal CBF, which was similar to the levels of CBF found in aged people [7]. The $2 \mathrm{VO}$ induced $\mathrm{CBF}$ reduction could be divided into 3 phases: acute, chronic and restitution phases. In the acute phase, during a couple of days after $2 \mathrm{VO}$ 
onset, $\mathrm{CBF}$ was rapidly reduced around $66.7 \%$ of the control in the corpus callosum and around $21.6 \%$ in the hippocampus $[8,9]$. In the chronic phase, CBF gradually increased but still could not reach the normal levels. The duration of the chronic phase was around 2 weeks to 3 months [7]. The pathology found in this phase was similar to ageing, including white matter lesions, neuronal death in the hippocampal CA1 subregion and cognitive impairments $[7,10]$. The restitution phase was the period after 6 months of ischemia, and $\mathrm{CBF}$ in this phase returned to the normal levels [7]. Chronic cerebral hypoperfusion in rats induced neuronal loss in the hippocampal areas, lesions of the white matter in the corpus callosum and internal capsule, leading to spatial learning and memory impairment [11].

Inflammation is one of the main causes of pathogenesis in chronic cerebral hypoperfusion. Several pro-inflammatory cytokines released from activated microglia, injured neurons and reactive astrocytes have critical roles in neuronal damage. They produced inducible nitric oxide synthase (iNOS) and other pro-inflammatory cytokines such as tumor necrosis factor alpha (TNF- $\alpha$ ) and interleukin-1beta (IL-1 $\beta$ ), resulting in inflammatory responses. In the ischemic condition, iNOS caused a substantial production of nitric oxide (NO) which reacted rapidly with superoxide anion radicals and generated peroxynitrite molecules which were cytotoxic [12]. The increased expression of iNOS contributed to the pathophysiology of cerebral hypoperfusion due to the activation of inflammatory downstream cascades. Cilostazol, which scavenged hydroxyl and peroxyl radicals, was given in $2 \mathrm{VO}$ rats for 14 days after $2 \mathrm{VO}$ induction. The results showed that cilostazol reduced apoptotic cell death, TNF- $\alpha$ positive cells, GFAP-positive cells and white matter lesions in the optic tract, leading to the attenuation of spatial learning and memory impairment in $2 \mathrm{VO}$ animals [13]. However, cilostazol and other free radical scavengers e.g. edaravone are chemical substances and have short-term and long-term side effects in humans such as nausea, vomiting, serious allergic reaction, headache, palpitation, loose stool and diarrhea [14].

Thai Herbal Sahatsatara formula (STF), a polyherbal mixture, has been traditionally prescribed for muscle pain and numbness in Thailand for a long time. This formula was registered in the National List of Essential Medicine (NLEM) 2011 of Thailand. STF consists of 21 medicinal ingredients: Anacyclus pyrethrum (L.), Anethum graveolens L., Arorus calamus L., Atractylodes lancea,
Baliospermum montanum, Cinamomum camphora, Cuminum cyminum L., Ferula assafoetida L., Kleinhovia hospita L., Lepidium sativum L., Merremia vitifolia, Myristica fragrans Houtt. (fruit), Myristica fragrans Houtt.(mace), Nigella sativa L., Picrorrhiza kurroa Kutaki, Pimpinella anisum L., Piper nigrum L., Piper retrofractum Vahl., Plumbago indica L., Terminalia chebula Retz. (fruit), Terminalia chebula Retz. (gall) and synthetic camphor. Previous studies have shown that STF possessed antioxidant and anti-inflammatory activities. STF significantly inhibited reactive oxygen species (ROS) production in primary human dermal fibroblasts (NHDFs) after the induction by IL-1 $\beta$ [15]. The $95 \% \mathrm{EtOH}$ extract of STF could inhibit COX-2 expression and TNF- $\alpha$ production induced by lipopolysaccharide in murine macrophages (RAW246.7) [16]. Moreover, piperine, an alkaloid group from $P$. nigrum and $P$. indica [15], is the main active ingredient in STF. Intraperitoneal injections of piperine $(0.1$ and $0.5 \mathrm{mg} / \mathrm{kg} /$ day) were shown to attenuate spatial learning and memory impairment in the Morris water maze task and to reduce oxidative stress on day 5 after the 2VO [17]. Piperine also showed antioxidant activity, enhanced neuronal density in the hippocampus [11] and had neuroprotective effects through the regulation of apoptotic proteins in rats with middle cerebral artery occlusion [18].

Other ingredients in STF also demonstrated beneficial effects in the cognitive functions. Oral administration of Myristica fragrans extract ( $5 \mathrm{mg} / \mathrm{kg}$ ) for 3 days enhanced memory in aged mice in the elevated plus-maze task. This dose of Myristica fragrans extract could also reverse scopolamineand diazepam-induced impairment in learning and memory [19]. Other components of STF such as Plumbago indica L., Terminalia chebula Retz, Myristica fragrans Houtt. and Nigella sativa L. also had antioxidant potentials, enhancing learning and memory in normal rodents $[19,20]$. Moreover, the ingredients such as Acorus calamus L., Lepidium sativum L., Ferula assa-foetida Regel (gum)., Nigella sativa L., Atractylodes lancea Thunb. DC and Picrorhiza kurroa Royle ex Benth. had vasodilatation and hemodynamic effects [21], which might provide additional effects in the event of chronic cerebral hypoperfusion.

In summary, these ingredients in STF have shown beneficial neuroprotective effects which are anti-inflammation, antioxidant, vasodilation, and hemodynamic effects in both in vitro and in vivo experiments. Chronic cerebral hypoperfusion causes the reduction of cerebral blood flow, leading to the 
inflammation and oxidative stress which are the main pathophysiology resulting in the brain injury. However, the protective effect of STF has never been investigated in a model of chronic cerebral hypoperfusion before. Thus, this study aimed to determine whether STF could attenuate the pathophysiological changes induced by chronic cerebral hypoperfusion in middle-aged rats.

\section{MATERIALS AND METHODS}

\section{Animals}

Twelve months old male Wistar rats (450-500 g) were acquired from the National Laboratory Animal Center, Mahidol University Salaya Campus, Nakornpathom, Thailand. They were kept in ventilated isolation cages with constant room temperature $\left(23^{\circ} \mathrm{C} \pm 2{ }^{\circ} \mathrm{C}\right)$ under the natural light/dark cycle. The animals could freely access to the standard diet and water. Experimental protocols were approved by Siriraj Animal Care and Use Committee, Faculty of Medicine Siriraj Hospital, Mahidol University, Thailand (SI-ACUP 007/2560). The rats were habituated for 2 weeks before the initiation of the experiments.

\section{Preparation of Sahatsatara formula (STF)}

All herbal and mineral ingredients were characteristically authenticated by 2 Applied Thai traditional specialists (Table S1). The crude drug powder of STF was produced by the Manufacturing Unit of Herbal Medicines and Products Ayurved Siriraj under Good Manufacturing Practice (GMP) PIC/S regulation (Lot number 21206-004Y). The certification and registration number of STF were G265/52 certified by FDA of Thailand with the recommended oral dose of 1.0-1.5 gm before meals, 3 times per day. The chemical determination was examined by thin-layer chromatography and high-performance liquid chromatography $[15,21]$. The fingerprint chromatograms and content determination of piperine in STF was determined (Figs. S1 and S2). Crude powder of STF has dark brown color and pungent taste [21] with the percentage of piperine in the powder approximately $0.602 \%(\mathrm{w} / \mathrm{w})$ (Table S2). The crude powder of STF was suspended in sterile water.

\section{Drug administration}

Rats in the Sham+SW group received sterile water. $2 \mathrm{VO}$ rats were randomly assigned into 3 groups: $2 \mathrm{VO}+\mathrm{SW}$ group receiving sterile water, 2 VO+STF300 group receiving STF $(300 \mathrm{mg} / \mathrm{kg}$ bw) and 2VO+STF1000 group receiving STF $(1000 \mathrm{mg} / \mathrm{kg} \mathrm{bw})$. STF powder was suspended in 3-7 $\mathrm{ml}$ of sterile water, starting with $3 \mathrm{ml}$ of saline water; however, the volume was increased up to $7 \mathrm{ml}$ in high doses in which the administrations were split into 2 times of $3.5 \mathrm{ml}$ suspension with the 15-min interval. The STF suspensions were freshly prepared every day and the administrations were conducted at around 10 am daily by gavage.

\section{Experimental design}

In order to examine the action of STF against chronic cerebral hypoperfusion induced by $2 \mathrm{VO}$, 28 rats ( $n=7$ per group) were randomly allocated into 4 groups which were Sham+SW, 2VO+SW, 2VO+STF300 and 2VO+STF1000. Rats in each group started receiving either STF or sterile water orally after $2 \mathrm{VO}$ induction for 3 days and continued daily until the end. All rats were carried out for spatial learning and memory test by the Morris water maze (MWM) task after the $2 \mathrm{VO}$ induction for 60 days. At the end of the behavioral experiment, the brains were removed and collected for the investigations of numbers of hippocampal neurons and white matter injury in the corpus callosum.

\section{Surgical procedure}

All rats were anesthetized with an intraperitoneal injection of ketamine $(60 \mathrm{mg} / \mathrm{kg})$ and xylazine $(0.6 \mathrm{mg} / \mathrm{kg})$ mixture [22]. After the rats were unconscious, the neck region was cleansed with $70 \%$ alcohol. A small incision was made at the midline of the neck region. In the $2 \mathrm{VO}$ protocol, bilateral common carotid arteries (CCA) were separated from the cervical sympathetic and vagal nerves. The right CCA was permanently ligated first with 4-0 type surgical silk. Then, the left CCA was permanently ligated. The skin and soft tissues were sutured. In the sham group, the rats received the same surgical procedure without the ligation.

\section{Morris water maze (MWM) task}

Spatial learning and memory test in the MWM task were carried out in all rats after the surgery for $2 \mathrm{VO}$ induction for 60 days ( $n=7$ per group). The protocol was slightly modified from the previous study [22]. The visible platform trial was used to assess the motivation to escape, visual ability and locomotor activity of the rats. It was done only on the first day of the training. In the acquisition trial conducted $24 \mathrm{hr}$ after the visible platform trial, the rats were allowed to swim for $120 \mathrm{~s}$ to find the hidden platform. Escape latencies to find the 
hidden platform of each rat were recorded 4 sessions per day for 7 days with 30-min inter-trial intervals between each session. The acquisition probe trial was performed on the next day after the acquisition trial. In this trial, the platform was removed from the pool, and the rats were allowed to swim for 120 s. Time spent in the target quadrant, which was used to evaluate the ability to retain previously acquired spatial memory, was recorded. In the reversal trial conducted $24 \mathrm{~h}$ later, the platform was placed in the quadrant opposite to the target quadrant in the acquisition trial. The rats were trained consecutively for 7 days in order to evaluate the intellectual ability to adapt to a new situation or spatial learning flexibility. Lastly, the reversal probe trial was performed on the last day of the MWM task. The swimming sessions of the reversal trial and the reversal probe trial were similar to the acquisition trial and the acquisition probe trial, respectively. The swimming speeds were calculated from the first session of the visible platform trial to evaluate locomotor activity.

\section{Histological assessments}

Twenty-four hours after the reversal probe trial (after the surgery for $2 \mathrm{VO}$ induction for 77 days), the rats were deeply anesthetized with an overdose of intraperitoneal injection of ketamine $(180 \mathrm{mg} / \mathrm{kg})$ and xylazine $(1.8 \mathrm{mg} / \mathrm{kg})$ mixture. After being unconscious, the rats were sacrificed by transcardial perfusion. The histological preparation was modified from Koomhin et al [22]. Coronal sections were cut at $10 \mu \mathrm{m}$ by using a microtome (Leica RM2255 rotary microtome, USA). The first set of the sections was stained with $0.1 \%$ cresyl violet for investigating the numbers of surviving neurons in the hippocampus. The stained slides were examined under a light microscope (Carl Zeiss Axio Imager M2, Germany). Pictures of the hippocampus were taken under the microscope at 200X magnification for surviving neuron counting. For each brain, series of 3 sections spaced at an interval of $100 \mu \mathrm{m}$ from each other were analyzed. For each slide, the total numbers of surviving neurons in an area of $530 \times 700 \mu \mathrm{m}^{2}$ (approximately $0.4 \mathrm{~mm}^{2}$ ) of the hippocampal CA1, CA3 and dentate gyrus (DG) were counted by an investigator who was blind to the experimental groups. The surviving neurons were identified by their large nuclei with prominent nucleoli.

The second set of the sections was stained with Luxol fast blue for white matter analysis in the corpus callosum. The photographs of the corpus callo- sum were taken under a light microscope (Carl Zeiss Axio Imager M2, Germany) at 400X magnification. Areas of the corpus callosum were blindly analyzed. The severity of white matter injuries was graded by 2 independent examiners as follows: normal (grade 0); disarrangement of nerve fibers (grade 1); formation of marked vacuoles (grade 2); and disappearance of myelinated fibers (grade 3) [23]. The 3 representative sections were examined.

\section{Statistical analysis}

Statistical analysis was conducted with SPSS 17.0. All results were expressed as mean \pm SEM. The differences of escape latency and time spent in the target quadrant from the MWM task were analyzed by repeated measures ANOVA followed by LSD post hoc test. The numbers of surviving neurons in all subregions of the hippocampus and grading scores of white matter injuries were analyzed by one-way ANOVA followed by LSD post hoc test for pairwise comparison. The statistical significance was accepted at $p<0.05$.

\section{RESULTS}

\section{Effects of STF on spatial learning and memory performance}

The escape latencies and swimming speeds in the visible trial were not significantly different between each experimental group (Fig. 1), indicating that the visual and locomotor abilities and motivation to escape of the rats were not affected by chronic cerebral hypoperfusion.

Permanent bilateral CCA occlusion induced impairments in the spatial learning and spatial memory retention in the acquisition trial and the acquisition probe trial, respectively. Moreover, chronic cerebral hypoperfusion also induced deficits in learning flexibility in the reversal trial and in memory retention of a newly acquired memory in the reversal probe trial. The treatment with STF (1000 mg/kg) could attenuate the impairments in the spatial learning and learning flexibility in the acquisition trial and the reversal trial, respectively.

The escape latencies of the $2 \mathrm{VO}+\mathrm{SW}$ group were significantly longer than that of the Sham+SW group on day $1,4,5$, and $6(p<0.05)$ of the acquisition trial. The escape latency of the 2VO+STF1000 group was shorter than that of $2 \mathrm{VO}+\mathrm{SW}$ group with a significant difference on day $4(p<0.05)$ (Fig. 2a). These results indicated that chronic cerebral hypoperfusion for 60 days induced spatial learning and memory deficit, and 


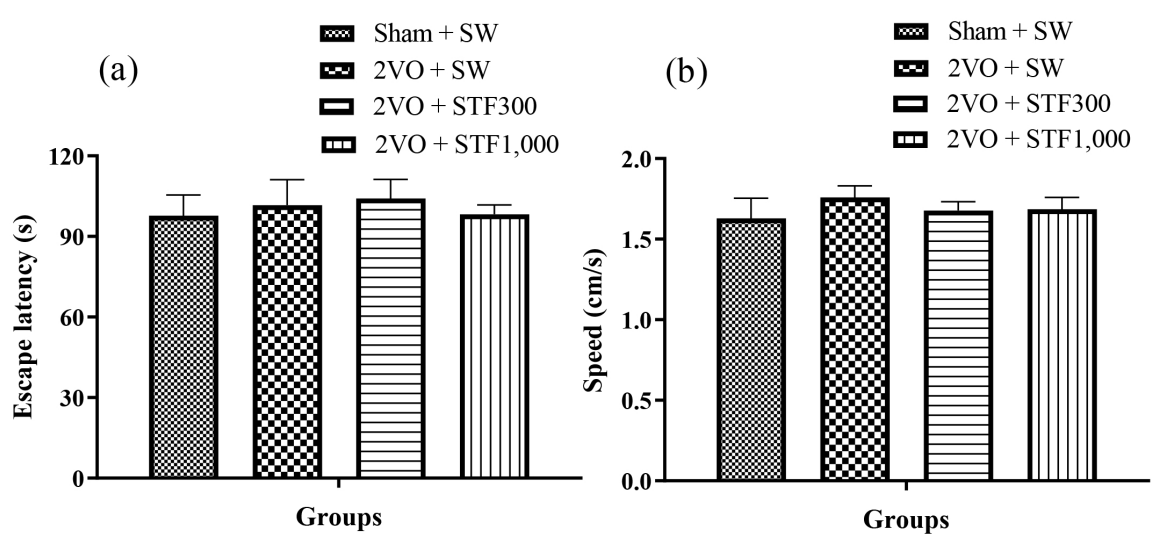

Fig. 1 Effect of STF on the visual and locomotor activities. Escape latencies in the visible platform trial (a) and swimming speeds from the first session of the visible platform trial (b) at day 60 after 2VO induction. Data are expressed as mean \pm SEM, $n=7$ per group.
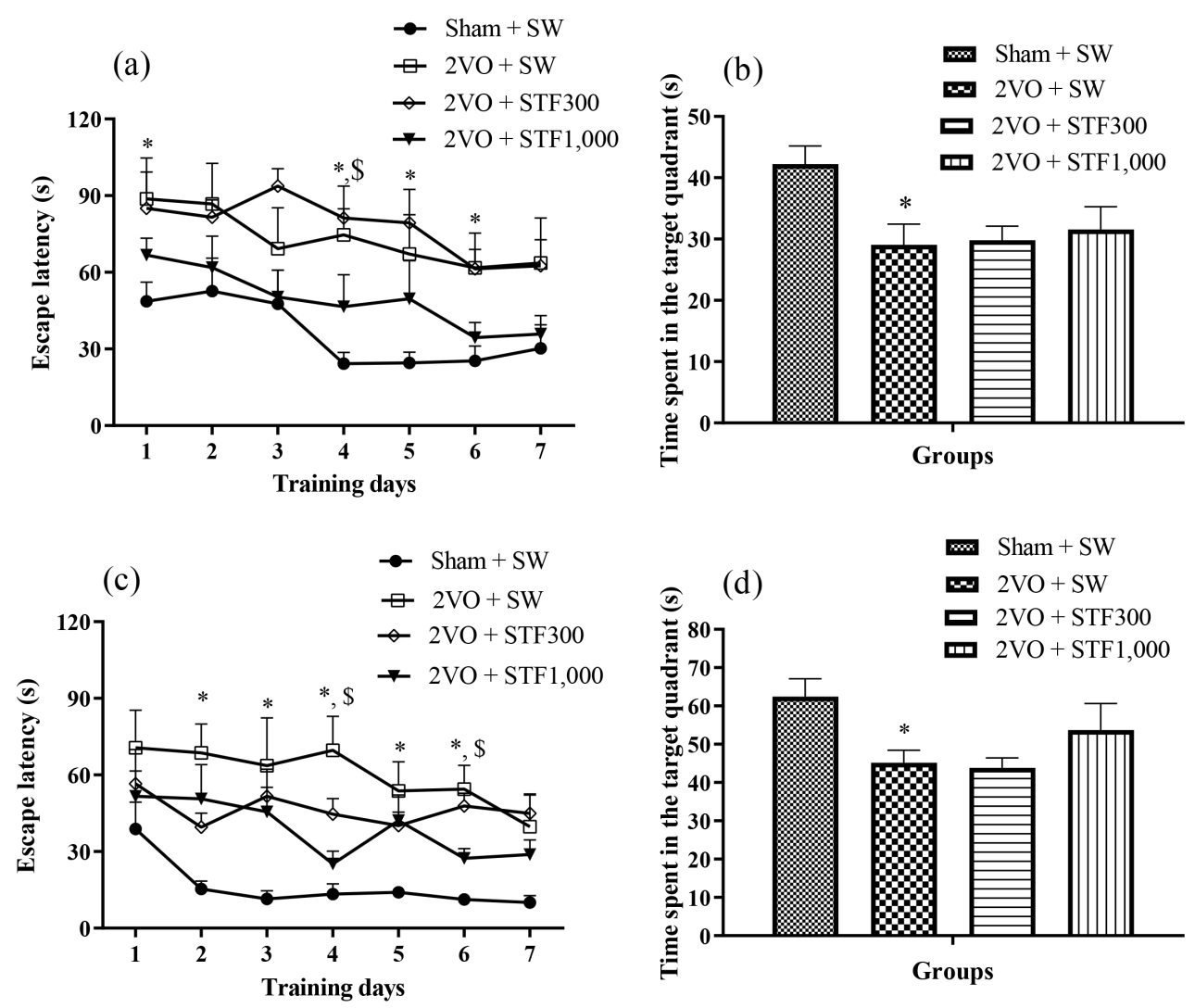

Fig. 2 Effect of STF on spatial learning and memory performance. (a) The escape latencies after 60 days of $2 \mathrm{VO}$ represent daily spatial learning performance in the acquisition trial day 1 to day 7; (b) the time spent in the target quadrant in the acquisition probe trial represents memory retention; (c) the escape latencies in the reversal trial represent learning flexibility performance; and (d) the time spent in the target quadrant in the reversal probe trial indicates the ability to retain newly acquired memories. Data are expressed in mean \pm SEM of 7 animals per group. $\$ p<0.05$ compared between the 2VO+STF1000 and 2VO+SW groups. ${ }^{*} p<0.05$ compared between the $2 \mathrm{VO}+\mathrm{SW}$ and Sham+SW groups. 
Table 1 Effects of STF on the numbers of surviving neurons ( $n=5$ per group) in the hippocampus.

\begin{tabular}{lccc}
\hline \multirow{2}{*}{ Group } & \multicolumn{3}{c}{ Surviving neuron $\left(\right.$ cell $\left./ 0.4 \mathrm{~mm}^{2}\right)$} \\
\cline { 2 - 4 } & CA1 & CA3 & DG \\
\hline Sham+SW & $77.65 \pm 7.04$ & $76.86 \pm 5.86$ & $153.28 \pm 10.40$ \\
2VO+SW & $59.96 \pm 8.28$ & $76.73 \pm 6.10$ & $140.71 \pm 9.29$ \\
2VO+STF300 & $61.98 \pm 4.06$ & $73.98 \pm 9.62$ & $145.92 \pm 8.62$ \\
2VO+STF1000 & $83.63 \pm 6.45 *$ & $79.96 \pm 5.94$ & $164.91 \pm 4.67$ \\
\hline
\end{tabular}

Data are expressed in mean \pm SEM; * $p<0.05$ compared to $2 \mathrm{VO}+\mathrm{SW}$.

STF $(1000 \mathrm{mg} / \mathrm{kg}$ ) could attenuate the deficit induced by $2 \mathrm{VO}$. The retention of spatial memory was tested in the acquisition probe trial. The $2 \mathrm{VO}+\mathrm{SW}$ group exhibited a significant reduction in the time spent in the target quadrant when compared to the Sham+SW group $(p<0.05)$, but there was no significant difference between the 2VO+STF300 and $2 \mathrm{VO}+\mathrm{SW}$ groups or between the $2 \mathrm{VO}+\mathrm{STF} 1000$ and $2 \mathrm{VO}+\mathrm{SW}$ groups (Fig. 2b). The results indicated that $2 \mathrm{VO}$ induced chronic cerebral hypoperfusion also caused an impairment in the retention of spatial memory; however, STF treatment could not significantly attenuate the impairment.

In the reversal trial, the escape latencies of the $2 \mathrm{VO}+\mathrm{SW}$ group were significantly longer than those of the Sham $+\mathrm{SW}$ group on day $2,3,4$, 5 and $6(p<0.05)$. The escape latencies of the 2VO+STF1000 group were significantly shorter than those of the $2 \mathrm{VO}+\mathrm{SW}$ group on day 4 and 6 $(p<0.05)$ (Fig. 2c). In the reversal probe trial, there was a significant difference in the time spent in the target quadrant between the $2 \mathrm{VO}+\mathrm{SW}$ and Sham + SW groups $(p<0.05)$. The time spent in the target quadrant of the $2 \mathrm{VO}+\mathrm{SW}$ group was not significantly different from all STF treated groups (Fig. 2d). These results demonstrated that 2VO induced chronic cerebral hypoperfusion caused the deficits in the reversal trial and the reversal probe trial, indicating the impairments of learning flexibility and retention of newly acquired memory. STF at the dose of $1000 \mathrm{mg} / \mathrm{kg}$ could attenuate the impairment in the learning flexibility but not in the retention of newly acquired memory.

\section{Effects of STF on the numbers of surviving hippocampal neurons}

The numbers of surviving neurons in each subregion of the hippocampus were determined after the end of the MWM task (77 days after the 2VO induction). The numbers of surviving neurons in the left and the right subregions were averaged and shown as averaged values in each subregion. The numbers of surviving neurons among 4 groups (Sham+SW, $2 \mathrm{VO}+\mathrm{SW}, 2 \mathrm{VO}+\mathrm{STF} 300$ and $2 \mathrm{VO}+\mathrm{STF} 1000$ ) were not significantly different in all subregions of the hippocampus (CA1, CA3 and dentate gyrus) (Fig. 3 and Table 1). The results indicated that chronic cerebral hypoperfusion in 12-month-old rats did not significantly affect the numbers of surviving hippocampal neurons although the behavioral deficits had been clearly shown.

\section{Effects of STF on white matter injury}

This study investigated the changes of white matter at the corpus callosum after the end of the MWM task. After $2 \mathrm{VO}$ induction for 77 days, the $2 \mathrm{VO}+\mathrm{SW}$ group had a significantly higher white matter injury grading score than the Sham+SW group $(p<0.05)$. The grading score of the 2VO+STF1000 group was significantly lower than that of the $2 \mathrm{VO}+\mathrm{SW}$ group ( $p<0.05$ ) (Fig. 4). These results indicated that chronic cerebral hypoperfusion in 12-month-old rats significantly caused white matter injury in the corpus callosum, a major communication pathway between 2 brain hemispheres. Treatment with STF at the dose of $1000 \mathrm{mg} / \mathrm{kg}$ significantly reduced white matter injury induced by $2 \mathrm{VO}$.

\section{DISCUSSION}

Chronic cerebral hypoperfusion was proposed to induce cognitive impairment by causing white matter lesions and neuronal damage at the CA1 subregion of the hippocampus, which was vulnerable to ischemia [24]. The present study also demonstrated cognitive impairment after 60 days of chronic cerebral hypoperfusion, but we found only mild neuronal damage at the hippocampal CA1 subregion. However, white matter lesions were clearly observed in the corpus callosum.

Permanent bilateral common carotid artery occlusion (2VO) is a classical model to mimic mild cognitive deficit in aged people. In adult rats, 2VO leads to moderate cerebral blood flow (CBF) reduction in the forebrain $[5,8,9,25]$. During the $2 \mathrm{VO}$ induced cerebral hypoperfusion, the pathophysiology found during the chronic phase is similar to the pathologies occurring in elderly people. Cerebral hypoperfusion causes an increase of proinflammatory mediators and ROS, leading to white matter lesions and loss of pyramidal neurons especially in the CA1 subregion of the hippocampus, and eventually leading to cognitive impairments [26].

Middle-aged male Wistar rats at 12 months of age were used in this study, and the cognitive 

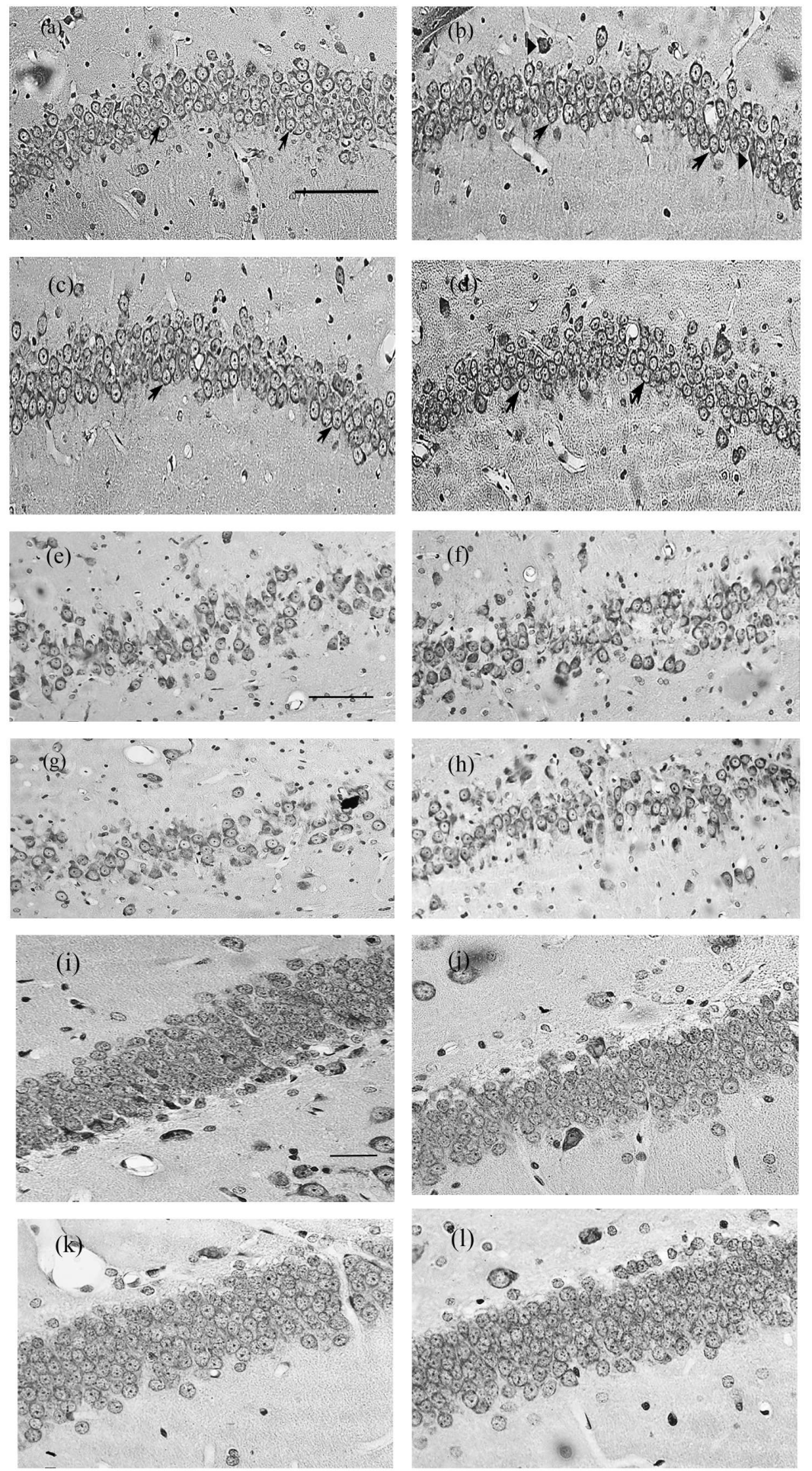

Fig. 3 Effects of STF on the numbers of surviving neurons. Photographs show representative cresyl violet staining of neurons in different subregions of the hippocampus. Panels ((a)-(d)) represent the CA1 subregion of the Sham+SW, 2VO+SW, 2VO+STF300 and 2VO+STF1000 groups, respectively. Surviving neurons show large nuclei with prominent nucleoli (arrow) while dead neurons show the cell shrinkage with pyknotic appearance (arrowhead). Scale bar $=$ $100 \mu \mathrm{m}$ (magnification $=200 \mathrm{X}$ ). Panels $((\mathrm{e})-(\mathrm{h})$ ) represent the CA3 subregion. Scale bar $=100 \mu \mathrm{m}$ (magnification $=$ 200X). Panels ((i)-(1)) represent the dentate gyrus. Scale bar $=50 \mu \mathrm{m}$ (magnification $=400 \mathrm{X}$ ). 

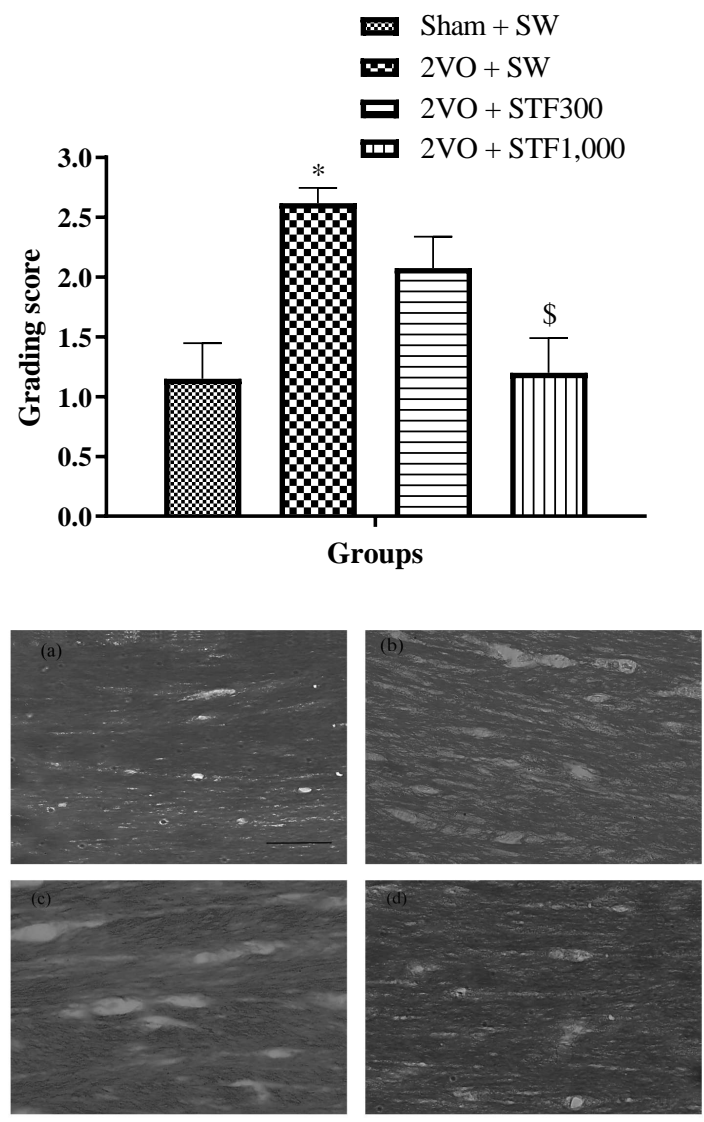

Fig. 4 Effects of STF on white matter injury in the corpus callosum. Photographs show representative Luxol fast blue staining ((a)-(d)) of the Sham+SW, 2VO+SW, $2 \mathrm{VO}+\mathrm{STF} 300$ and $2 \mathrm{VO}+\mathrm{STF} 1000$ groups, respectively. White matter injury grading scores are expressed in mean \pm SEM of 5 animals per group. ${ }^{*} p<0.05$ compared to the Sham+SW group. $\$ p<0.05$ compared to the $2 \mathrm{VO}+\mathrm{SW}$ group. Scale bar $=100 \mu \mathrm{m}$ (magnification $=$ 400X).

impairment was induced by $2 \mathrm{VO}$ for 60 days. All rats with chronic cerebral hypoperfusion showed no deficits in visual and locomotor activities and motivation to escape. The results were consistent with the findings of other studies $[10,22]$. The rats which suffered from chronic cerebral hypoperfusion showed longer escape latencies in the acquisition trial and the reversal trial of the MWM task than the sham group, indicating the deterioration in learning performance and learning flexibility, respectively. Time spent in the target quadrant of the $2 \mathrm{VO}$ group was shorter than that of the sham group both in the acquisition probe trial and the reversal probe trial, suggesting a deficit in spatial memory retention. These results indicated that chronic cerebral hypoperfusion caused impairments in spatial learning, spatial learning flexibility and spatial memory retention. The behavioral results in this study were in accordance with the previous studies, demonstrating that chronic cerebral hypoperfusion caused an impairment in spatial learning and memory $[5,10,27]$.

The energy metabolism was greatly affected in the acute phase of cerebral hypoperfusion due to the marked reduction of CBF. However, the energy metabolism of the $2 \mathrm{VO}$ group was not significantly altered from the sham group after 3 weeks of chronic cerebral hypoperfusion [28], and the CBF returned to the baseline levels between 8 weeks to 3 months $[9,25]$. These results suggested that the sufficient $\mathrm{CBF}$ in the chronic phase may be supplied from the collateral pathways via compensatory mechanisms such as a dilation in the vertebrobasilar system and angiogenesis, perhaps leading to neurogenesis in the degenerated hippocampal CA1 subregion after the cerebral ischemia was induced [29]. In young rats with age ranging between 6 weeks to 3 months, the neuronal loss in the hippocampus was clearly observed from 1 week to 11 weeks of cerebral hypoperfusion [10,30,31]. However, a study using 12-month-old male Wistar rats similar to the age range of our study reported that the rats with 2VO induced cerebral hypoperfusion for 21 days did not show neuronal loss in the CA1 subregion of the hippocampus and cerebral cortex [28]. These results were similar to our data in which 12-monthold rats with 77 days of cerebral hypoperfusion were used, and the findings demonstrated that there was no significant difference in the numbers of surviving neurons among 4 groups in any hippocampal subregions. Furthermore, studies which used rats at 9 months of age demonstrated that they did not observe neuronal cell loss in the CA1 subregion of the hippocampus at 30 days but observed at 120 days after $2 \mathrm{VO}$ induction [4]. Pappas et al [5] studied male Sprague-Dawley rat retired breeders at 9-10 months of age and reported that rats with chronic cerebral hypoperfusion for 190 days showed neuronal loss in the CA1 subregion of the hippocampus but did not show the loss at 14 days of $2 \mathrm{VO}$. The evidence suggested a delayed observation of neuronal loss in the hippocampal subregions in the middle-aged animals with chronic cerebral hypoperfusion when compared with the loss in young animals. The delayed observation may be due to a delayed progression of neuronal loss or an addition 
of new neurons due to angiogenesis.

However, the remaining hippocampal neurons might not be able to rescue spatial learning and memory deficits because of an impairment in synaptic functions. Studies showed that the expression of the microtubule associated protein-2 (MAP-2), a cytoskeletal phosphoprotein associated with microtubules and postsynaptic densities, and synaptophysin protein, a marker for presynaptic terminals, in the hippocampus was significantly decreased at 8 weeks [32], 10 weeks and 20 weeks [27] after chronic cerebral hypoperfusion induction. Furthermore, several studies showed marked spatial learning and memory impairment even though mild neuronal loss was observed in the CA1 subregion of the hippocampus $[5,10]$. A study has demonstrated that spatial learning and memory impairment was still observed at 6 months after $2 \mathrm{VO}$ induction, although CBF returned to the normal [10]. The results indicated that the numbers of hippocampal neurons did not correlate well with learning and memory performance. The spatial learning and memory were impaired even though the number of hippocampal neurons was not different from the sham group. The cognitive impairment might be a result from the changes in the structures or numbers of dendritic spines.

More importantly, the present study showed substantial white matter lesions after 77 days of $2 \mathrm{VO}$. The white matter receives blood supply from the penetrating arteries. They are end arteries which will be markedly affected by a reduction of CBF [33], making the white matter vulnerable to cerebral ischemia $[2,31]$. White matter lesion is one of the causes of cognitive function deficits in aged people [34]. The lesions of white matter caused disconnection among the prefrontal cortices and other brain regions, resulting in the reduction of prefrontal cortex activation and leading to cognitive impairment [35]. The corpus callosum has the highest density of white matter fibers that interconnect between 2 hemispheres. The white matter in the corpus callosum was chosen as a representation of the global white matter in this study. White matter lesions in our study might be correlated with the impairment in learning flexibility in the reversal trial of the MWM task. This data suggested that the hippocampal and dorsolateral prefrontal circuits might be disrupted. This finding was consistent with a study in aged people demonstrating the association between age-associated white matter lesions and cognitive flexibility deficits [35]. However, the mechanism of white matter degeneration is still unclear. The migration of leukocytes into the brain parenchyma resulted from the breakdown of BBB after cerebral hypoperfusion might cause white matter lesions [36]. There were studies reporting that BBB breakdown was observed in the middleaged rats (12 months of age) [37] and old rats (24 months of age) [38,39]. After 1 hour of $2 \mathrm{VO}$ induction, activated microglia appeared in the white matter and were still observed and peaked at day 7 to day 14. The number of GFAP-positive cells increased in the white matter after 7 days of $2 \mathrm{VO}$ [40]. This data indicated that microglia, astrocytes and leukocytes were stimulated in the early phase of cerebral hypoperfusion. These cells produced proinflammatory mediators (e.g. TNF- $\alpha$, IL- $1 \beta$, iNOS and COX-2) and ROS which deteriorate the white matter [40]. White matter lesions might be the main cause in the impairment of learning flexibility induced by chronic cerebral hypoperfusion in the middle-aged rats.

Administration of STF at the dose of $1000 \mathrm{mg} / \mathrm{kg}$ could attenuate white matter lesions at the corpus callosum. The present study demonstrated that 12-month-old rats with $2 \mathrm{VO}$ showed the same white matter lesions as aged humans. This study is the first study to investigate the effects of STF on spatial learning and memory in chronic cerebral hypoperfused rats. Administration of STF at the dose of $300 \mathrm{mg} / \mathrm{kg}$ did not show the protective effect in chronic cerebral hypoperfused rats. However, administration of STF at the dose of $1000 \mathrm{mg} / \mathrm{kg}$ showed the attenuation of spatial learning impairment in the acquisition trial and the attenuation of learning flexibility impairment in the reversal trial. Furthermore, STF at the dose of $1000 \mathrm{mg} / \mathrm{kg}$ could also significantly protect white matter lesions in the corpus callosum from chronic cerebral hypoperfusion induced by $2 \mathrm{VO}$. Nonetheless, it seems that the ameliorating effect of STF $(1000 \mathrm{mg} / \mathrm{kg})$ was greater on the learning flexibility (2 days of significant effect in the reversal trial) than the spatial learning (1 day of significant effect in the acquisition trial). Since the flexibility to adjust the behavior to the new location of platform (new goal) or learning flexibility is closely related to the white matter function, so the better behavioral results in the reversal platform trial of MWM might support the protective effect of STF against the white matter injuries on learning flexibility. The protective effects of STF might come from piperine, the main active compound, that possesses antioxidant and anti-inflammation activities through the inhibition of ROS, TNF- $\alpha$ production 
and COX-2 expression $[15,16]$. Other ingredients in STF also have antioxidant, anti-inflammation, vasodilation and hemodynamic effects [21]. These activities might act cooperatively to reduce the inflammation and oxidative stress which are the main pathophysiology causing the brain injury and cognitive impairments. However, to elucidate the exact mechanisms underlying the protective effect of STF and how each ingredient in STF interacts to each other, the future investigations are required.

\section{CONCLUSION}

The administration of Sahatsatara at the dose of $1000 \mathrm{mg} / \mathrm{kg}$ for 60 consecutive days after 2VO induction could attenuate spatial learning and learning flexibility impairments. The effects might be due to the reduction of the white matter injury. These results indicated that Sahatsatara at the dose of $1000 \mathrm{mg} / \mathrm{kg}$ could protect white matter from chronic cerebral hypoperfusion. Therefore, it might be one of therapeutic choices in the attenuation of cognitive decline in aged people or vascular dementia patients.

\section{Appendix A. Supplementary data}

Supplementary data associated with this article can be found at http://dx.doi.org/10.2306/ scienceasia1513-1874.2020.084.

Acknowledgements: This research was supported by Siriraj Graduate Scholarship and Siriraj Research Fund (SRF) type 1, Faculty of Medicine Siriraj Hospital, Mahidol University, Thailand, Grant Number (IO) R016131013. We would like to thank Ms. Namphung Thongta, our research assistant, for her help in many aspects during the experimental period. This study would not have been achieved without her help.

\section{REFERENCES}

1. Ohnishi T, Hoshi H, Nagamachi S, Jinnouchi S, Flores LG 2nd, Futami S, Watanabe K (1995) Highresolution SPECT to assess hippocampal perfusion in neuropsychiatric diseases. J Nucl Med 36, 1163-1169.

2. Pantoni L, Garcia JH, Gutierrez JA (1996) Cerebral white matter is highly vulnerable to ischemia. Stroke 27, 1641-1647.

3. Gunning-Dixon FM, Brickman AM, Cheng JC, Alexopoulos GS (2009) Aging of cerebral white matter: a review of MRI findings. Int J Geriatr Psychiatry 24, 109-117.

4. Ni J, Ohta H, Matsumoto K, Watanabe H (1994) Progressive cognitive impairment following chronic cerebral hypoperfusion induced by permanent occlusion of bilateral carotid arteries in rats. Brain Res $\mathbf{6 5 3}$ 231-236.
5. Pappas BA, de la Torre JC, Davidson CM, Keyes MT, Fortin T (1996) Chronic reduction of cerebral blood flow in the adult rat: late-emerging CA1 cell loss and memory dysfunction. Brain Res 708, 50-58.

6. de la Torre JC (2004) Is Alzheimer's disease a neurodegenerative or a vascular disorder? Data, dogma, and dialectics. Lancet Neurol 3, 184-190.

7. Farkas E, Luiten PG, Bari F (2007) Permanent, bilateral common carotid artery occlusion in the rat: a model for chronic cerebral hypoperfusionrelated neurodegenerative diseases. Brain Res Rev 54, 162-180.

8. Tsuchiya M, Sako K, Yura S, Yonemasu Y (1992) Cerebral blood flow and histopathological changes following permanent bilateral carotid artery ligation in Wistar rats. Exp Brain Res 89, 87-92.

9. Otori T, Katsumata T, Muramatsu H, Kashiwagi F, Katayama Y, Terashi A (2003) Long-term measurement of cerebral blood flow and metabolism in a rat chronic hypoperfusion model. Clin Exp Pharmacol Physiol 30, 266-272.

10. Cechetti F, Pagnussat AS, Worm PV, Elsner VR, Ben J, da Costa MS, Mestriner R, Weis SN, et al (2012) Chronic brain hypoperfusion causes early glial activation and neuronal death, and subsequent long-term memory impairment. Brain Res Bull 87, 109-116.

11. Chonpathompikunlert P, Wattanathorn J, Muchimapura S (2010) Piperine, the main alkaloid of Thai black pepper, protects against neurodegeneration and cognitive impairment in animal model of cognitive deficit like condition of Alzheimer's disease. Food Chem Toxicol 48, 798-802.

12. Mracsko E, Hugyecz M, Institoris A, Farkas E, Bari F (2010) Changes in pro-oxidant and antioxidant enzyme levels during cerebral hypoperfusion in rats. Brain Res 1321, 13-19.

13. Watanabe $\mathrm{T}$, Zhang N, Liu M, Tanaka R, Mizuno Y, Urabe T (2006) Cilostazol protects against brain white matter damage and cognitive impairment in a rat model of chronic cerebral hypoperfusion. Stroke 37, 1539-1545.

14. Sorkin EM, Markham A (1999) Cilostazol. Drugs Aging 14, 63-73.

15. Thamsermsang $\mathrm{O}$, Akarasereenont $\mathrm{P}$, Laohapand $\mathrm{T}$, Panich U (2017) IL-1 beta-induced modulation of gene expression profile in human dermal fibroblasts: the effects of Thai herbal Sahatsatara formula, piperine and gallic acid possessing antioxidant properties. BMC Complem Altern M 17, 32-44.

16. Kakatum N, Jaiarree N, Makchucit S, Itharat A (2012) Antioxidant and anti-inflammatory activities of Thai medicinal plants in Sahasthara remedy for muscle pain treatment. J Med Assoc Thai 95S1, S120-S126. 
17. Pensirinapa S, Unchern S (2003) Benificial effects of piperine on spatial memory impairment and brain lipid peroxidation increase induced by transient cerebral ischemia in mice. Thai J Pharmacol 25, ID 82.

18. Hua S, Liu J, Zhang Y, Li J, Zhang X, Dong L, Zhao Y, Fu X (2019) Piperine as a neuroprotective functional component in rats with cerebral ischemic injury. Food Sci Nutr 7, 3443-3451.

19. Parle M, Dhingra D, Kulkarni SK (2004) Improvement of mouse memory by Myristica fragrans seeds. J Med Food 7, 157-161.

20. Tamadonfard Z, Sepehrara L, Jovhary H (2014) The effect of nigella sativa extract on learning and spatial memory of adult male rats. J Jahrom Univ Med Sci 12, 27-36.

21. Booranasubkajorn S, Huabprasert S, Wattanarangsan J, Chotitham P, Jutasompakorn P, Laohapand T, Akarasereenont P, Tripatara P (2017) Vasculoprotective and vasodilatation effects of herbal formula (Sahatsatara) and piperine in spontaneously hypertensive rats. Phytomedicine 24, 148-156.

22. Koomhin P, Tilokskulchai K, Tapechum S (2012) Ceftriaxone improves spatial learning and memory in chronic cerebral hypoperfused rats. ScienceAsia 38, 356-363.

23. Wakita H, Tomimoto H, Akiguchi I, Kimura J (1995) Protective effect of cyclosporin A on white matter changes in the rat brain after chronic cerebral hypoperfusion. Stroke 26, 1415-1422.

24. Tomimoto H, Ihara M, Wakita H, Ohtani R, Lin JX, Akiguchi I, Kinoshita M, Shibasaki H (2003) Chronic cerebral hypoperfusion induces white matter lesions and loss of oligodendroglia with DNA fragmentation in the rat. Acta Neuropathol 106, 527-534.

25. Ohta $H$, Nishikawa $H$, Kimura $H$, Anayama $H$, Miyamoto M (1997) Chronic cerebral hypoperfusion by permanent internal carotid ligation produces learning impairment without brain damage in rats. Neuroscience 79, 1039-1050.

26. Miyamoto N, Maki T, Pham LD, Hayakawa K, Seo $\mathrm{JH}$, Mandeville ET, Mandeville JB, Kim K, et al (2013) Oxidative stress interferes with white matter renewal after prolonged cerebral hypoperfusion in mice. Stroke 44, 3516-3521.

27. Liu HX, Zhang JJ, Zheng P, Zhang Y (2005) Altered expression of MAP-2, GAP-43, and synaptophysin in the hippocampus of rats with chronic cerebral hypoperfusion correlates with cognitive impairment. Brain Res Mol Brain Res 139, .

28. Plaschke K, Grant M, Weigand MA, Zuchner J, Martin E, Bardenheuer HJ (2001) Neuromodulatory effect of propentofylline on rat brain under acute and longterm hypoperfusion. Br J Pharmacol 133, 107-116.

29. Salazar-Colocho P, Lanciego JL, Del Rio J, Frechilla D (2008) Ischemia induces cell proliferation and neurogenesis in the gerbil hippocampus in response to neuronal death. Neurosci Res 61, 27-37.

30. Schmidt-Kastner R, Aguirre-Chen C, Saul I, Yick L, Hamasaki D, Busto R, Ginsberg MD (2005) Astrocytes react to oligemia in the forebrain induced by chronic bilateral common carotid artery occlusion in rats. Brain Res 1052, 28-39.

31. Xiong Z, Liu C, Wang F, Li C, Wang W, Wang J, Chen J (2006) Protective effects of breviscapine on ischemic vascular dementia in rats. Biol Pharm Bull 29, 1880-1885.

32. Zhu H, Zhang J, Sun H, Zhang L, Liu H, Zeng X, Yang Y, Yao Z (2011) An enriched environment reverses the synaptic plasticity deficit induced by chronic cerebral hypoperfusion. Neurosci Lett 502, 71-75.

33. Moody DM, Bell MA, Challa VR (1990) Features of the cerebral vascular patternthat predict vulnerability to perfusion or oxygenation deficiency: an anatomic study. AJNR Am J Neuroradiol 11, 431-439.

34. Wardlaw JM, Allerhand M, Doubal FN, Hernandez MV, Morris Z, Gow AJ, Bastin M, Starr JM, et al (2014) Vascular risk factors, large-artery atheroma, and brain white matter hyperintensities. Neurology 82, 1331-1338.

35. Nordahl CW, Ranganath C, Yonelinas AP, Decarli C, Fletcher E, Jagust WJ (2006) White matter changes compromise prefrontal cortex function in healthy elderly individuals. J Cogn Neurosci 18, 418-429.

36. Pantoni L (2002) Pathophysiology of age-related cerebral white matter changes. Cerebrovasc Dis 13S2, 7-10.

37. Takechi R, Pallebage-Gamarallage MM, Lam V, Giles C, Mamo JC (2013) Aging-related changes in bloodbrain barrier integrity and the effect of dietary fat. Neurodegener Dis 12, 125-135.

38. Elahy M, Jackaman C, Mamo JC, Lam V, Dhaliwal SS, Giles C, Nelson D, Takechi R (2015) Blood-brain barrier dysfunction developed during normal aging is associated with inflammation and loss of tight junctions but not with leukocyte recruitment. Immun Ageing 12, 1-9.

39. De Reuck JL (2012) Histopathological stainings and definitions of vascular disruptions in the elderly brain. Exp Gerontol 47, 834-837.

40. Wakita H, Tomimoto H, Akiguchi I, Kimura J (1994) Glial activation and white matter changes in the rat brain induced by chronic cerebral hypoperfusion: an immunohistochemical study. Acta Neuropathol 87, 484-492. 


\section{Appendix A. Supplementary data}

Table S1 The components of Thai Herbal Sahatsatara formula (STF) and its ratio in the formula.

\begin{tabular}{rlcc}
\hline No. & Scientific name & Part of usage & Ratio (\% w/w) \\
\hline 1 & $1,7,7$ - trimethylbicyclo (2.2.1) hepta-2-one (Synthetic camphor) & Crystal & 1.4 \\
2 & Acorus calamus L. & Root & 8.8 \\
3 & Anethum graveolens L. & Seed & 1 \\
4 & Atractylodes lancea (Thunb.) DC. & Root & 0.5 \\
5 & Baliospermum solanifolium (Burm.) Suresh & Root & 8 \\
6 & Clausena excavate Burm.f. & Stem & 4.8 \\
7 & Cuminium cyminum L. & Seed & 0.8 \\
8 & Ferula assa-foetida Regel & Gum & 1 \\
9 & Lepidium sativum L. & Seed & 1.1 \\
10 & Merremia vitifolia (Burm.f.) Hallier.f. & Stem & 0.8 \\
11 & Myristica fragrans Houtt. & Mace & 1.3 \\
12 & Myristica fragrans Houtt. & Fruit & 1.2 \\
13 & Nigella sativa L. & Seed & 0.7 \\
14 & Pistacia chinensis subsp. integerrima(J.L. Stewart ex Brandis) Rech. f. Lagasca & Root & 0.6 \\
15 & Picrorhiza kurroa Royle ex Benth. & Root & 0.4 \\
16 & Pimpinella anisum L. & Seed & 0.9 \\
17 & Piper nigrum L. & Fruit & 24 \\
18 & Piper retrofractum Vahl. & Fruit & 9.6 \\
19 & Plumbago indica L. & Root & 22.4 \\
20 & Terminalia chebula Retz. & Fruit & 10.4 \\
21 & Terminalia chebula Retz. & Gall & 0.3 \\
\hline
\end{tabular}

Table S2 The piperine contents in STF extract $(100 \mu \mathrm{g} / \mathrm{ml})$.

\begin{tabular}{|c|c|c|c|c|c|c|c|}
\hline Vial & Sample name & Name & RT & Area & High & Amount $(\mu \mathrm{g} / \mathrm{ml})$ & Amount (\% w/w) \\
\hline 1 & \multirow{3}{*}{ STF extract } & \multirow{3}{*}{ Piperine } & 5.197 & 157250 & 14993 & 3.065 & 0.606 \\
\hline 2 & & & 5.193 & 155071 & 15011 & 3.022 & 0.598 \\
\hline 3 & & & 5.192 & 155883 & 15045 & 3.038 & 0.601 \\
\hline & Mean & & 5.194 & 156068 & 15016 & 3.041 & 0.602 \\
\hline & Std. Dev. & & 0.003 & 1101 & 26.4 & 0.022 & 0.004 \\
\hline & $\%$ RSD & & 0.057 & 0.706 & 0.176 & 0.723 & 0.715 \\
\hline
\end{tabular}

$\mathrm{RT}=$ retention time $(\mathrm{min}) ;{ }^{*}(\mathrm{w} / \mathrm{w})=$ milligram unit in STF powder. 

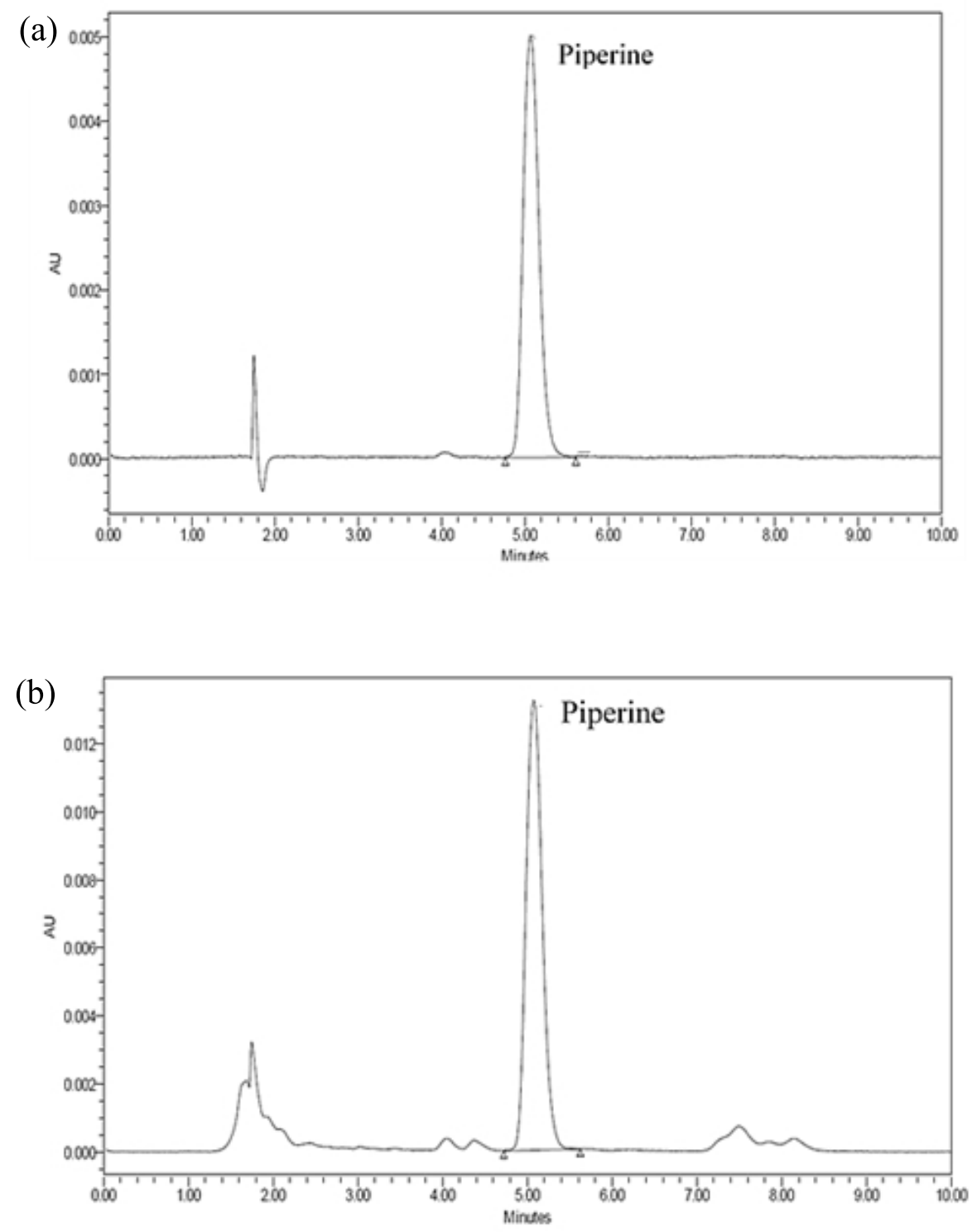

Fig. S1 (a) The RP-HPLC chromatogram of piperine standard $30 \mu \mathrm{g} / \mathrm{ml}$ and (b) piperine in STF extract $(100 \mu \mathrm{g} / \mathrm{ml})$. RP-HPLC $=$ reversed phrase-high performance liquid chromatography. 

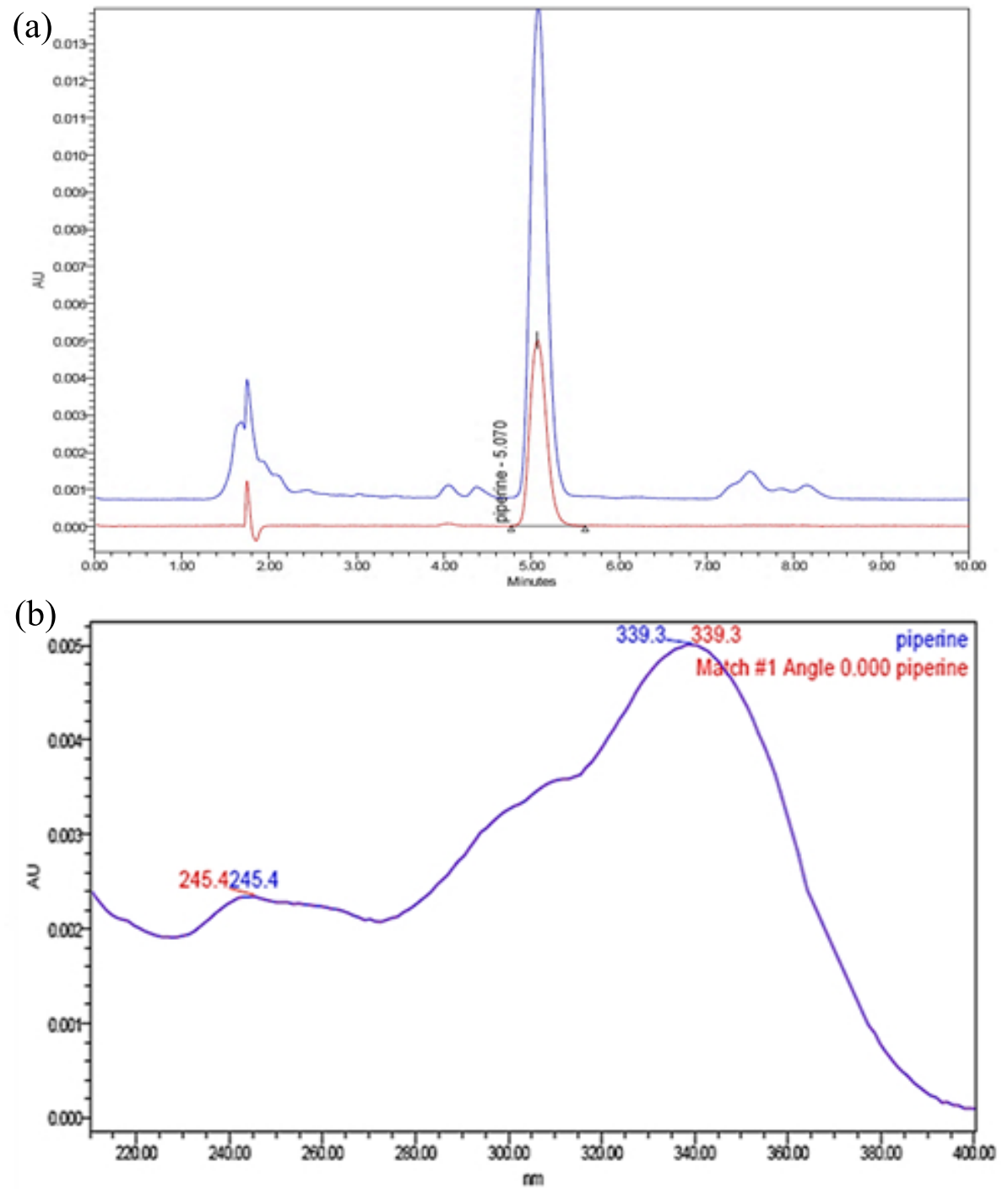

Fig. S2 (a) The overlay chromatograms and (b) the RP-HPLC matched UV absorbance pattern of piperine in STF extract (blue) represented the same pattern as piperine (red) at $340 \mathrm{~nm}$. RP-HPLC $=$ reversed phrase-high performance liquid chromatography. 